\title{
Probing chaos and biodiversity in a simple competition model
}

\author{
Lionel Roques ${ }^{\mathrm{a}, *}$ and Mickaël D. Chekroun ${ }^{\mathrm{b}}$ \\ ${ }^{a}$ UR 546 Biostatistique et Processus Spatiaux, INRA, F-84000 Avignon, France \\ b Environmental Research and Teaching Institute, École Normale Supérieure, \\ 24 rue Lhomond, 75231 Paris Cedex 05, France.
}

\begin{abstract}
Recent theoretical work has reported that chaos facilitates biodiversity. In this paper, we study the lowest-dimensional Lotka-Volterra competition model that exhibits chaotic trajectories, a model with four species. We observe that interaction and growth parameters leading respectively to extinction of three species, or coexistence of two, three or four species, are for the most part arranged in large regions with clear boundaries. Small islands of parameters that lead to chaos are also found. These regions where chaos occurs are, in the three cases presented here, situated at the interface between a non-chaotic four-species region and a region where extinction occurs. This implies a high sensitivity of biodiversity with respect to parameter variations in the chaotic regions. Additionally, in regions where extinction occurs which are adjacent to chaotic regions, the computation of local Lyapunov exponents reveals that a possible cause of extinction is the overly strong fluctuations in species abundances induced by local chaos at the beginning of the interval of study. For this model, we conclude that biodiversity is a necessary condition for chaos rather than a consequence of chaos, which can be seen as a signal of a high extinction risk.
\end{abstract}

Keywords: biodiversity; chaos; Lotka-Volterra; competition model; Lyapunov exponent; local Lyapunov exponent; simulated annealing

\section{Introduction}

Chaos was one of the great discoveries of the last century: The study of chaos has demonstrated that deterministic systems could be unpredictable in the sense that nearby identical states can tend to diverge at an exponential rate. Taking its roots in the deep and seminal work of Poincaré (1892) chaos was revealed and "experienced" in the historical paper of Lorenz (1963), where its system became one of the classic icons of modern nonlinear dynamics. Independently, Anosov (1963), Arnol'd (1966), and Smale (1967), followed by Ruelle and Takens (1971), to name a few, introduced the foundational concepts and tools that provided the appropriate theoretical context declaring chaos a science. Nowadays it is widely recognised that chaos plays an important role in complex systems such as climate dynamics or geophysical fluid dynamics (Ghil et al., 2008). Chaos also arises in the most simple population models (May, 1974), and although empirical observations of chaos in nature are rare, several examples suggest that some populations indeed exhibit chaotic dynamics (see Oksanen and Oksanen 1992; Costantino et

\footnotetext{
*Author for correspondence. Email: lionel.roques@avignon.inra.fr. Tel: +33 432722153. Fax: +33 432722182
} 
al. 1997; Turchin and Ellner 2000, for vole, lemming, and insect populations, and Benincà et al. 2008, for plankton communities).

In the meantime, biodiversity became one of the principal topics of ecology. Classically, two approaches can be used to study biodiversity: neutral theories (Bell, 2001; Hubbell, 2001) and competition models (MacArthur and Levins 1967; Levin 1970; May 1974; Gilpin 1975; May and Leonard 1975; Smale 1976; Huisman and Weissing 1999. See also, for example, Levins 1969; Durret and Levin 1994; Hanski and Gilpin 1996; Cantrell and Cosner 2003 for spatial competition models).

In this work, we focus on the second approach. The competitive exclusion principle (Hardin, 1960) predicts that $n$ species cannot coexist on fewer than $n$ resources, although, in some realistic situations, dozens of species can coexist on a few resources. This is known as the paradox of plankton (Hutchinson, 1961). Non-equilibrium dynamics (Armstrong and McGehee, 1980), and chaotic fluctuations in species abundances have been proposed as an answer to this paradox (Huisman and Weissing, 1999) supporting the idea that chaos can be favourable to biodiversity.

However, is this favourable influence of chaos a general rule in competition models? In this paper, we study whether, in competition models exhibiting chaotic and non-chaotic dynamics depending on interaction and growth parameters, biodiversity is less or more sensitive to parameter variations in the chaotic regions of the parameter space.

To deal with this question, we begin at the bottom of the hierarchy of mathematical models of biodiversity, in the famous class of competitive Lotka-Volterra models (Lotka, 1925, 1932; Volterra, 1931). For three or fewer interacting species, these models cannot have chaotic solutions (Hirsch, 1982, 1985, 1988, 1989, 1990, 1991). For five or more species, any type of complicated dynamics can occur (Smale, 1976). In the limit case of four species, Arnéodo et al. (1982) and, more recently, Vano et al. (2006) have demonstrated that chaotic dynamics can be exhibited. In that sense, the four-species Lotka-Volterra models are the simplest models of biodiversity that support chaotic dynamics. Here, the four species compete for four resources (see Armstrong and McGehee 1980, for an explanation); hence, the competitive exclusion principle does not apply. However, as we shall see, competitive exclusion can still occur. Indeed, extinction of one or more species occurs for a wide region in the parameter space.

For these four-species models, there are twenty determining parameters that render a surgical dissection of the parameter space practically impossible. However, we shall present a method that provides a partial but significant solution to the distribution of chaos, along with biodiversity, in the parameter space. To reach this goal, we use an appropriately selected twodimensional plane in the 20-dimensional space that intersects parameter regions where chaotic dynamics occur. Islands of chaos are thus exhibited as regions surrounded by regions leading to the extinction of three species, or the coexistence of two, three or four species. Unexpectedly well-organised structures of the parameter space are illustrated by this approach. The study of biodiversity is then enhanced by this organisation. 


\section{Materials and methods}

\subsection{The model}

Models for interacting species have been introduced by Lotka (1925) and Volterra (1931). The general form of these models is

$$
\frac{d N_{i}}{d t}=r_{i} N_{i}\left(1-\sum_{j=1}^{n} a_{i j} N_{j}\right), i=1 \ldots n,
$$

where $N_{i}$ denotes the population size of the $i^{\text {th }}$ species in proportion to its carrying capacity, $r_{i} \geq 0$ denotes its intrinsic growth rate, and $a_{i j}$ are intraspecific (if $i=j$ ) and interspecific (if $i \neq j$ ) interaction coefficients. Depending on the sign of the coefficients $a_{i j}$, system (1) can model predator-prey interactions, mutualism, or competition (Murray, 2002). In this paper, we focus on the situation where each species competes with each other species, corresponding to $a_{i j} \geq 0$, for all $i, j=1 \ldots n$. Furthermore, we assume that the intraspecific competition terms $a_{i i}$ are positive, and because the $N_{i}$ are expressed in terms of the carrying capacity, we get $a_{i i}=1$ for $i=1 \ldots n$. Under these assumptions, chaotic dynamics can be observed (Arnéodo et al., 1982) for $n \geq 4$. We place ourselves in the lowest-dimensional case, $n=4$.

\subsection{Measuring biodiversity}

In this model, self-organised dynamics can drive one or more species to extinction, as $t \rightarrow+\infty$. Several indices exist to measure biodiversity (e.g., Simpson's index and Shannon's index, which take an evenness criterion into account; i.e., the relative abundance of the different species). However, due to the small number of competing species, we choose to simply enumerate the persisting species over time instead. For our purpose, we say that a species does not persist if, after a certain time, it remains below an a priori fixed threshold $\varepsilon$.

\subsection{Probing chaos in the parameter space}

With a slight abuse of language, we can say that a point (resp. a region) in the parameter space is chaotic if, for some initial condition, the parameters corresponding to this point (resp. region) lead to chaotic trajectories.

To study the sensitivity of biodiversity with respect to parameter variations in chaotic regions compared to non-chaotic regions, we first have to find chaotic regions in the parameter space. As underscored by the work of Vano et al. (2006), the quest for these regions is challenging and requires powerful maximisation algorithms.

Classically, if the largest Lyapunov exponent of a system is positive, the system can be defined to be chaotic (see e.g. Eckmann and Ruelle, 1985). The Lyapunov exponents are the average exponential rates of divergence or convergence of nearby orbits in phase space. The magnitude of the exponent reflects the time scale on which system dynamics become unpredictable (Shaw, 1981). A stochastic minimisation algorithm applied to a well-chosen auxiliary functional (Appendix A), permits us to detect three points in the parameter space with positive positive Lyapunov exponents (by "Lyapunov exponent", we mean the largest Lyapunov exponent): $\left(\mathcal{R}_{1}, \mathcal{A}_{1}\right),\left(\mathcal{R}_{2}, \mathcal{A}_{2}\right)$ and $\left(\mathcal{R}_{3}, \mathcal{A}_{3}\right)$, where the $\mathcal{R}_{x}$ correspond to the growth vectors $\left(r_{i}\right)$, and the $\mathcal{A}_{x}$ correspond to the interaction matrices $\left(a_{i j}\right)$ in the model $(1)$. The corresponding Lyapunov exponents are $\lambda_{1}=0.04, \lambda_{2}=0.01$ and $\lambda_{3}=0.03$, respectively. See 
Fig. 1 for examples of trajectories $N(t)$ obtained with the above parameters. Exact parameter values of the three chaotic points are provided in Appendix A.

Remark 2.1 Thanks to the algorithm presented in Appendix A, we were able to build several chaotic points. Each successful run led to a different chaotic point, suggesting that, though rare in measure in the parameter space, there are numerous chaotic regions. The point $\left(\mathcal{R}_{1}, \mathcal{A}_{1}\right)$ was chosen close to the chaotic point presented by Vano et al. (2006), but with a higher Lyapunov exponent, whereas the two other chaotic points, $\left(\mathcal{R}_{2}, \mathcal{A}_{2}\right)$ and $\left(\mathcal{R}_{3}, \mathcal{A}_{3}\right)$, were chosen arbitrarily among the obtained chaotic points. Incidentally, in the planar section $\mathcal{P}$ of the parameter space, those three points belong to disconnected chaotic regions, showing that the set of chaotic points in the parameter space is not convex.

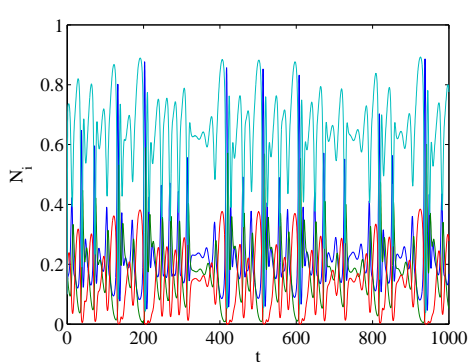

(a)

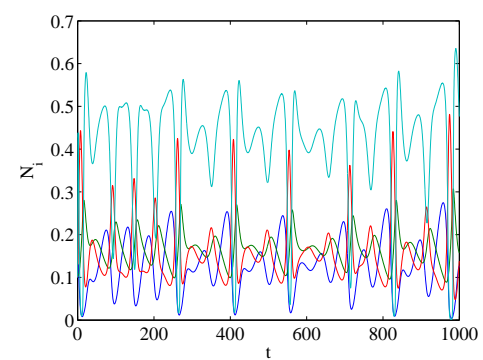

(b)

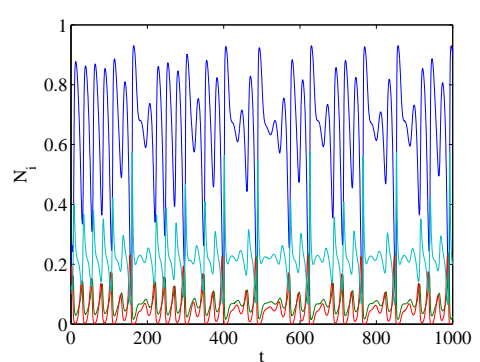

(c)

Figure 1: Chaotic trajectories $N(t)=\left[N_{1}(t) N_{2}(t) N_{3}(t) N_{4}(t)\right]$, obtained with parameters $\left(\mathcal{R}_{1}, \mathcal{A}_{1}\right)(\mathrm{a}),\left(\mathcal{R}_{2}, \mathcal{A}_{2}\right)(\mathrm{b})$, and $\left(\mathcal{R}_{3}, \mathcal{A}_{3}\right)(\mathrm{c})$, with the initial datum $N(0)=\left[\begin{array}{llll}0.1 & 0.1 & 0.1 & 0.1\end{array}\right]$.

\subsection{Structure of the parameter space with respect to biodiversity and chaos}

We analyse the planar portion $\mathcal{P}$ of the parameter space containing the three points $\left(\mathcal{R}_{x}, \mathcal{A}_{x}\right)$. The elements in the two-dimensional plane $\mathcal{P}$ are of the following type:

$$
(\mathcal{R}, \mathcal{A})=\left(\mathcal{R}_{1}, \mathcal{A}_{1}\right)+\alpha\left(\mathcal{R}_{2}-\mathcal{R}_{1}, \mathcal{A}_{2}-\mathcal{A}_{1}\right)+\beta\left(\mathcal{R}_{3}-\mathcal{R}_{1}, \mathcal{A}_{3}-\mathcal{A}_{1}\right),
$$

for all real numbers $\alpha, \beta$ such that the above conditions on $\left(a_{i j}\right)$ and $\left(r_{i}\right)$ are fulfilled.

For $1.2 \times 10^{6}$ elements sampled uniformly in $\mathcal{P}$, we computed the solution of (1), with random initial data $N(0) \in(0,1)^{4}$, sampled under a uniform law. The ending time for the computation was set to $T=4000$.

We fixed $\varepsilon=10^{-3}$, and defined a species $i$ as extinct at time $T$ if its abundance $N_{i}$ was smaller than this threshold for all times in $\left(\frac{3 T}{4}, T\right)$. Then, for each computed solution, we measured the number of persisting species at time $T$.

The Lyapunov exponent $\lambda$ associated with each parameter, and each initial datum $N(0)$, were computed whenever the number of coexisting species at time $T$ was equal to four (the Lyapunov exponent was computed at $t=10^{4}$ ). As mentioned previously, systems with three or fewer species cannot exhibit chaotic dynamics, and the computation of the Lyapunov exponent is therefore of less interest in those cases. 


\subsection{Basins of attraction}

In Section 2.3, a chaotic point was defined as a point $(\mathcal{R}, \mathcal{A})$ leading to chaotic trajectories, at least for some initial condition. Depending on the initial condition, to each point in the parameter space may be associated several asymptotic behaviours if two or more attractors are present. In such cases, each attractor has a basin of initial conditions that lead to that attractor.

In order to get some insight into the shape of these basins, we analysed the asymptotic behaviour of the solutions of (1) in terms of the initial condition $N(0)$. Our analysis was conducted at three chaotic points, $\left(\left(\mathcal{R}_{1}, \mathcal{A}_{1}\right),\left(\mathcal{R}_{2}, \mathcal{A}_{2}\right)\right.$ and $\left.\left(\mathcal{R}_{3}, \mathcal{A}_{3}\right)\right)$, and at three non-chaotic points, $(\mathcal{R}, \mathcal{A})$ (defined by $(2)$, with $(\alpha, \beta)=(0.93,0),(0.4,0.4)$ and $(0.6,0.6)$, respectively).

\section{Results}

On each pixel of Fig. 2, the maximum number of coexisting species obtained among the 13 sets of parameters and initial data corresponding to this pixel $\left(300 \times 300\right.$ pixels for $1.2 \times 10^{6}$ elements, each one corresponding to $1.2 \times 10^{6}$ couples $(\alpha, \beta)$ and $1.2 \times 10^{6}$ initial data $\left.N(0)\right)$ is represented. Thus, Fig. 2 depicts the maximum number of species that can survive at time $T$, for parameters in $\mathcal{P}$. Similarly, in pixels where parameters leading to four species were found, the maximum Lyapunov exponent obtained among those parameters is represented.

Regions of the plane $\mathcal{P}$ corresponding to different numbers of coexisting species are mostly arranged in large geometrical structures with sharp boundaries. The region corresponding to four species occupies a small proportion of $\mathcal{P}$ compared with the other regions.

Every type of region has common boundaries. Hence, infinitesimal changes in parameters can lead to a jump from one type of region to another. However, away from these boundaries, the probability that such a change in parameters could lead to a change in the number of coexisting species remains very low.

Remark 3.1 Some regions in Fig. 2 look like fractals. In fact, as the number of initial data per pixel (13 in the case presented here) is increased, the boundaries of these regions become sharper. Compare Fig. 2 with Fig. 3, which was computed with more initial data per pixel. The occurrence of isolated points with different natures than their surrounding regions seems mainly to be caused by the sensitivity of the outcome of competition (number of coexisting species) with respect to initial conditions (see Section 2.5 and e.g. Grebogi et al., 1987; Huisman and Weissing, 2001a).

\subsection{Occurrence of Chaos}

The three chaotic points $\left(\mathcal{R}_{x}, \mathcal{A}_{x}\right), x=1 \ldots 3$, of coordinates $(0,0),(1,0)$ and $(0,1)$ in $\mathcal{P}$ are embedded in chaotic regions, say $\mathcal{C}_{1}, \mathcal{C}_{2}$ and $\mathcal{C}_{3}$, respectively. These three regions are narrow compared to $\mathcal{P}$, and no other regions in $\mathcal{P}$ are found to be chaotic, emphasising the rarity of chaos in the parameter space.

The spatial structure of these three chaotic regions is detailed in Fig. 3. In all cases, chaotic regions are situated at the interface between a region with less than four species and a nonchaotic region where four species coexist: $\mathcal{C}_{1}$, is surrounded by 4 -species and 2-species regions; $\mathcal{C}_{2}$ by 4 -species and 3 -species regions, and $\mathcal{C}_{3}$ by 4 -species and 1 -species regions. The maximum largest Lyapunov exponents in these regions do not exceed $\lambda_{1}, \lambda_{2}$ and $\lambda_{3}$, respectively. 

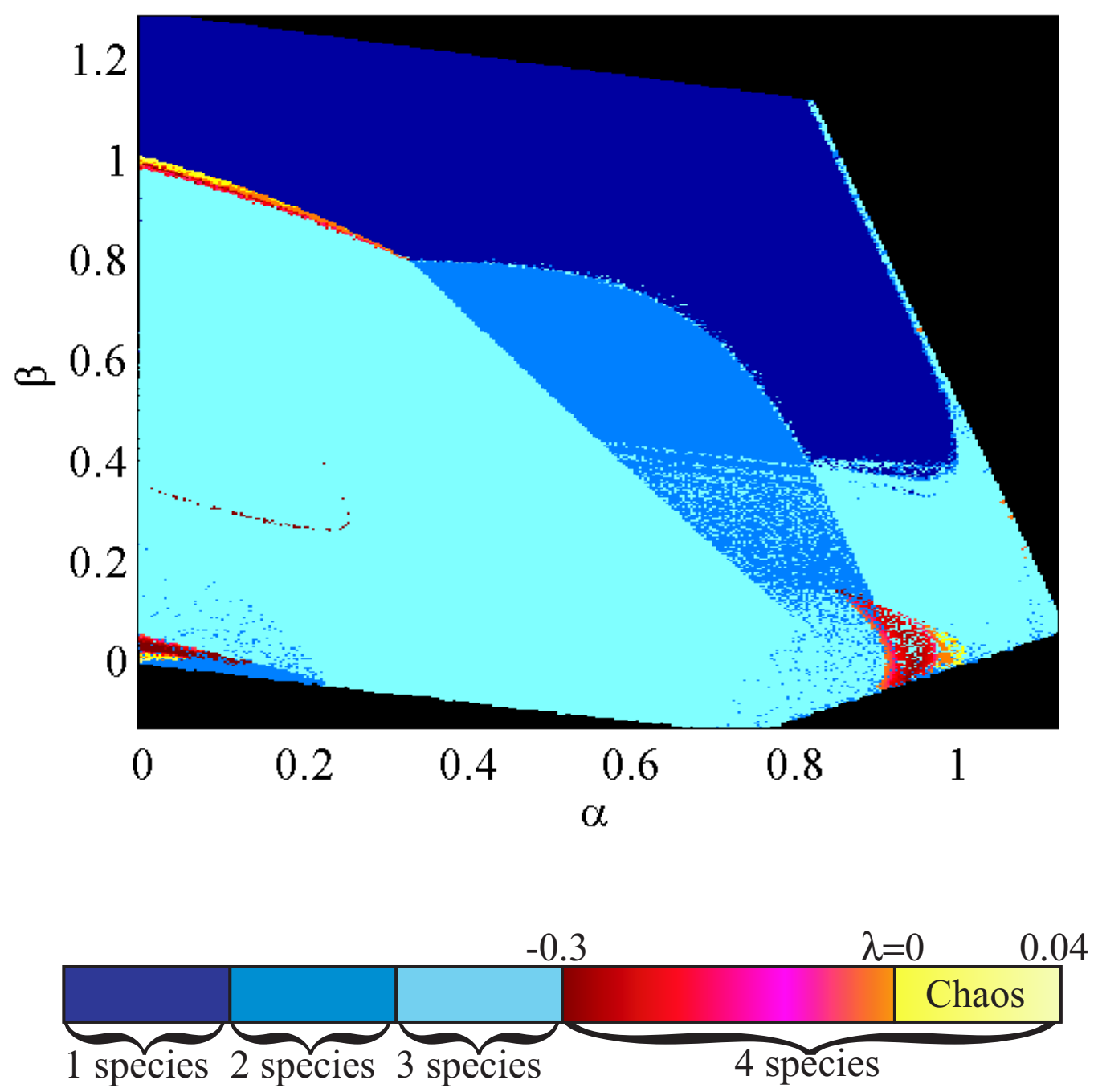

Figure 2: Occurrence of chaos and biodiversity in the planar section $\mathcal{P}$ of the parameter space. In this frame, the coordinates $(0,0),(1,0)$ and $(0,1)$ respectively correspond to the points $\left(\mathcal{R}_{1}, \mathcal{A}_{1}\right),\left(\mathcal{R}_{2}, \mathcal{A}_{2}\right)$ and $\left(\mathcal{R}_{3}, \mathcal{A}_{3}\right)$. The colours associated to each pixel of the figure correspond to the maximum computed number of coexisting species (resp. maximum Lyapunov exponent in four-species regions) for parameters in this pixel, over randomly sampled initial data $N(0)$. Black regions correspond to parameters outside $\mathcal{P}$. 


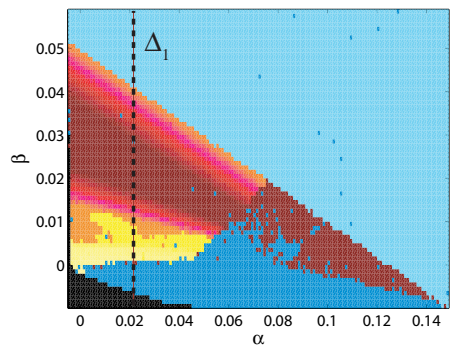

(a) $\mathcal{C}_{1}$

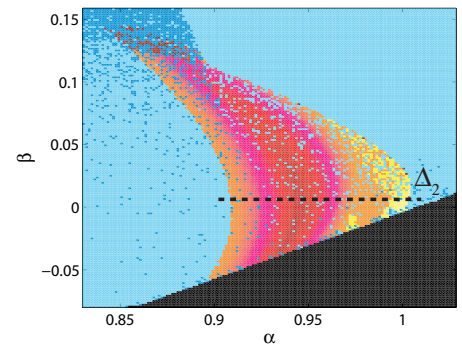

(b) $\mathcal{C}_{2}$

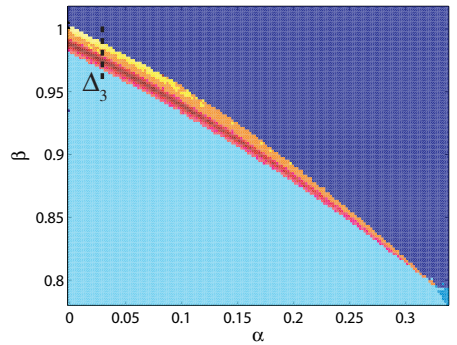

(c) $\mathcal{C}_{3}$

Figure 3: The three chaotic regions found in $\mathcal{P}$, and containing respectively the points $\left(\mathcal{R}_{1}, \mathcal{A}_{1}\right)$ (a), $\left(\mathcal{R}_{2}, \mathcal{A}_{2}\right)$ (b) and $\left(\mathcal{R}_{3}, \mathcal{A}_{3}\right)$ (c). Colour legend is the same as in Fig. 2. Computations were carried out with a finer resolution than in Fig. 2 and with other randomly chosen initial data $N(0)$. Those figures therefore do not correspond to exact magnifications of Fig. 2.

\subsection{Sudden biodiversity loss}

We now focus on the interface between chaotic and non-chaotic regions.

We computed the Lyapunov exponent on three segments $\Delta_{1}, \Delta_{2}, \Delta_{3}$ in $\mathcal{P}$, crossing each of the three chaotic regions (see Fig. 3, and Appendix B for the precise equations of these segments). The results are presented in Fig. 4. In all three cases, the route to chaos is comparable: $\lambda(\tau)$, seen as a function of its position $\tau$ on the segment, has a M-shaped profile with a soft transition to positive Lyapunov exponents via classical period doubling on one side of each segment (not presented here), and, on the other side, a sharp transition from positive values of $\lambda$ to negative values. An examination of Fig. 3 shows that these sharp transitions correspond to extinction of one species $\left(\Delta_{2}\right)$, two species $\left(\Delta_{1}\right)$ or three species $\left(\Delta_{3}\right)$. Fig. 4 shows that the highest values of Lyapunov exponents are found for parameter values near to the critical ones that lead to species extinction.

Conversely, the transition from non-chaotic to chaotic 4-species regions, and the transition from non-chaotic 4 -species regions to 3 -species regions, does not produce discontinuity in the Lyapunov exponent.

In the region where the function $\lambda(\tau)$ is positive, it exhibits strong oscillations, showing high sensitivity of chaotic strength with respect to parameter variations. Comparable oscillations can also be seen on $\Delta_{3}$ for negative values of $\lambda(\tau)$. In this last case, the trajectories $N(t) \in \mathbb{R}^{4}$ look chaotic for small times $t$ (not presented here), before one species is led to extinction. Such behaviour can be obtained when local Lyapunov exponents are positive for small times $t$.

The local Lyapunov exponent $\lambda_{t_{0}}$ measures the short-term (for $t<t_{0}$ ) average trajectory divergence rate (see e.g. Nese 1989; Ellner and Turchin 1995), whereas the Lyapunov exponent $\lambda=\lim _{t_{0} \rightarrow+\infty} \lambda_{t_{0}}$ is the long-term average divergence rate of nearby trajectories. A positive local Lyapunov exponent, for some time $t_{0}$, indicates "local chaos" (cf. Turchin 2003): small perturbations in trajectories, for times smaller than $t_{0}$, have large effects.

We computed local Lyapunov exponents $\lambda_{t_{0}}$ for parameters in $\Delta_{1}, \Delta_{2}$ and $\Delta_{3}$ (Fig. 4). In all cases, $\lambda_{t_{0}}$ remains positive near the interface where extinction occurs, and no discontinuity is observed. Furthermore, near this interface, $\lambda_{t_{0}}$ is higher for parameters leading to extinction than for parameters leading to chaotic trajectories. Those observations support the idea that overly strong local chaos, characterised by high values of the local Lyapunov exponent, leads to the extinction of one or more species, and subsequently to a sharp decrease in the Lyapunov 
exponent.

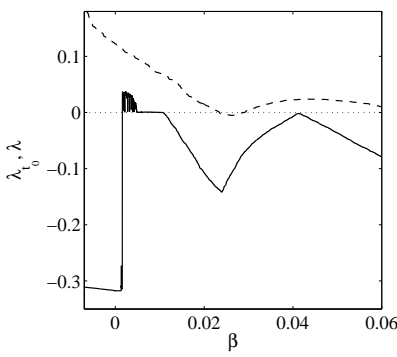

(a) $\Delta_{1}$

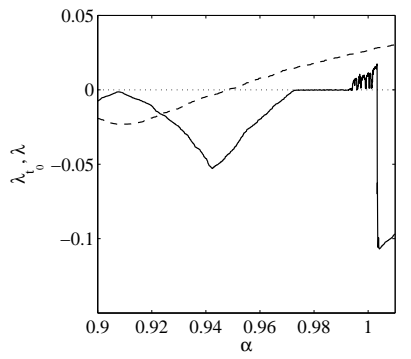

(b) $\Delta_{2}$

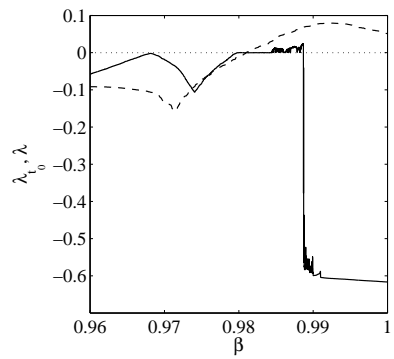

(c) $\Delta_{3}$

Figure 4: Lyapunov exponent $\lambda$ (plain lines), and local Lyapunov exponent $\lambda_{t_{0}}$ (dashed lines) computed over the segments $\Delta_{1}$ (a), $\Delta_{2}$ (b) and $\Delta_{3}$ (c), with the initial condition $N(0)=$ $\left[\begin{array}{llll}0.1 & 0.1 & 0.1 & 0.1\end{array}\right]$, and with $t_{0}=20, t_{0}=20$ and $t_{0}=25$ respectively in (a), (b) and (c).

\subsection{The role of the initial condition}

The above results focused on the effects of the parameters. For fixed parameter values, we now focus on the effect of the initial condition.

At each considered point (see Section 2.5), several attractors have been found; i.e., cases in which the asymptotic behaviour of $N(t)$ depends on $N(0)$. Depending on $N(0)$, the three chaotic points $\left(\left(\mathcal{R}_{1}, \mathcal{A}_{1}\right),\left(\mathcal{R}_{2}, \mathcal{A}_{2}\right)\right.$ and $\left.\left(\mathcal{R}_{3}, \mathcal{A}_{3}\right)\right)$ can lead-by definition-to chaos, or to the extinction of one or more species. The number of surviving species also depends on $N(0)$ at the three non-chaotic points tested here. Thus, in any case, biodiversity does depend on the initial state of the system. Fig. 5 depicts the behaviour of $N(t)$, at the chaotic point $\left(\mathcal{R}_{3}, \mathcal{A}_{3}\right)$, and at the non-chaotic point $(\alpha, \beta)=(0.93,0)$ in terms of $N(0)$.

The proportion of the basin of attraction of chaotic trajectories in the set of initial conditions $[0,1]^{4}$ can vary greatly between different chaotic points: this proportion is $0.1,0.03$, and 0.6 , respectively, at the points $\left(\mathcal{R}_{1}, \mathcal{A}_{1}\right),\left(\mathcal{R}_{2}, \mathcal{A}_{2}\right)$ and $\left(\mathcal{R}_{3}, \mathcal{A}_{3}\right)$. It is noteworthy that chaos can occur with significant probability at some points in the parameter space. In particular, the above observation implies that, for random initial conditions in $[0,1]^{4}$, chaos occurs with probability 0.6 at the point $\left(\mathcal{R}_{3}, \mathcal{A}_{3}\right)$.

Remark 3.2 We observe in Fig. 2.5 that the basin boundaries look like fractals. This observation holds at the six chaotic and non-chaotic tested points (see Section 2.5 for the definition of these points). This fractal structure of the basin boundaries is in contrast with the rather smooth structure of the regions of the parameter space corresponding to different numbers of coexisting species. As emphasised in Grebogi et al. (1987), fractal basin boundaries represent an obstruction to predictability: if there is an uncertainty in the initial condition, it may be very difficult to determine in which basin it lies.

\section{Discussion and conclusions}

We have analysed the structure of the interaction and growth parameter space, with respect to biodiversity and chaos, for the lowest-dimensional Lotka-Volterra competition model that may lead to chaotic trajectories; i.e., the model with four species. 


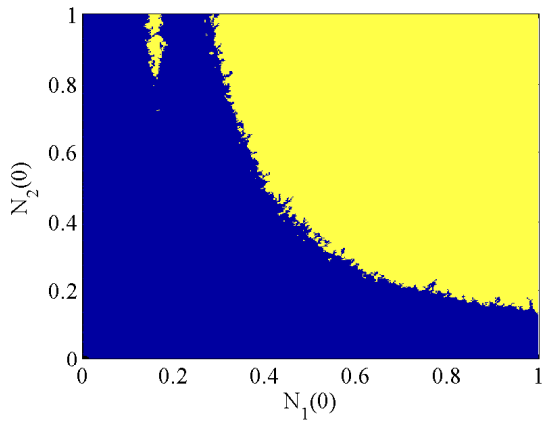

(a)

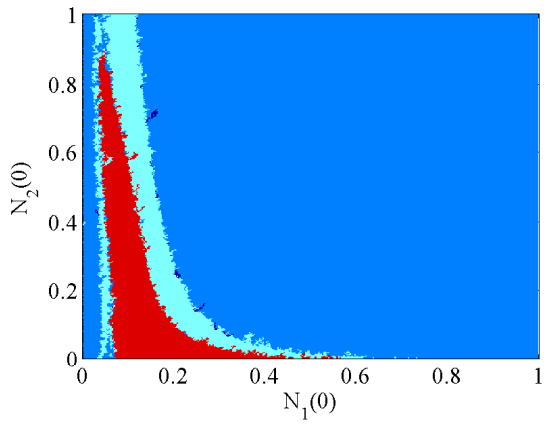

(b)

Figure 5: Occurrence of chaos and biodiversity in terms of the initial condition at the chaotic point $(\mathcal{R}, \mathcal{A})=\left(\mathcal{R}_{3}, \mathcal{A}_{3}\right)(\mathrm{a})$; and at the non-chaotic point defined by $(\alpha, \beta)=(0.93,0)$ (b). For our computations, we fixed $N_{3}(0)=N_{4}(0)=0.5$. Colour legend is the same as in Fig. 2 .

First, we observe the geometric structure of the parameter space with respect to the number of coexisting species. As emphasised by Vano et al. (2006), chaos occurs in narrow regions of the parameter space. We observe a recurring disposition of these regions with respect to other non-chaotic regions. In the three cases studied here, the parameters leading to chaos are indeed situated at the interface between a region where only three or fewer species can coexist, and a non-chaotic region where four species coexist. Thus, chaotic regions are not entirely surrounded by four-species regions.

Because of this disposition of chaotic regions with respect to the regions where extinction occurs, obtaining chaotic trajectories may indicate a risk for biodiversity. Small and even continuous variations in the interaction or growth parameters may indeed lead chaotic fourspecies systems to suddenly lose one or more species. Note that, as indicated in Fig. 4, non-chaotic four-species systems can also slowly shift from equilibrium to periodic $(\lambda=0)$ and chaotic dynamics, through continuous changes in the parameters. The occurrence of chaos is also strongly conditioned by the initial condition at time 0 .

What are the causes behind the sudden losses of biodiversity observed at the interface with chaotic regions? In chaotic regions, the highest Lyapunov exponents are found near this interface. Obviously, after this interface the Lyapunov exponent becomes negative. However, this does not exclude local chaos. Indeed, our computations reveal that local Lyapunov exponents keep on growing through this interface. This implies even higher local chaos in the regions where extinction occurs, compared with chaotic regions, and suggests that overly strong local chaos drives some species to extinction. As stochastic demography is often proposed as an explanation for species extinctions in discrete populations (Lande, 1993), the strong fluctuations in population abundances associated with local chaos would be, in our case, the cause of the species extinctions observed at interfaces with chaotic regions.

Our conclusions regarding the effects of chaos may seem in opposition to recent theory (Huisman and Weissing 1999; Huisman et al. 2001; Huisman and Weissing 2001b. See also Petrovskii and Malchow 2001, for spatio-temporal models). Indeed, in those works, chaos has been found to be a favourable factor that may enhance species biodiversity. More precisely, in a resource competition model, where $n$ species compete for $k$ resources $(k<n)$, Huisman and Weissing (1999) have shown that chaotic fluctuations in species abundance allows the coexistence of many species on few resources, which may be an answer to the paradox of 
plankton.

In fact, our results are not contradictory with the results in (Huisman and Weissing, 1999), which were obtained with a fixed set of parameters. Here, we address the issue of biodiversity sensitivity with respect to parameter variations, and we find higher sensitivity in chaotic regions compared with randomly chosen regions in the parameter space. Obviously, in our case, chaos is also associated with regions of high diversity, as chaotic trajectories cannot exist with fewer than four species. This does not mean that chaos is a favourable factor for species biodiversity, but simply that biodiversity is a necessary condition for chaos to arise.

The model studied in this paper is the simplest competitive model of ordinary differential equations that exhibits chaotic dynamics. A similar study should be conducted, for instance for the competition models treated in (Huisman and Weissing, 1999), to assess whether the unfavourableness of chaos observed in our work remains true in other settings.

\section{Appendix A: simulated annealing algorithm}

Our objective is to build an algorithm for finding parameters $R=\left(r_{i}\right)$ and $A=\left(a_{i j}\right)$, for $1 \leq i, j \leq n$, of system (1), leading to chaotic solutions. This problem can be reduced to a maximization problem, through the computation of the Lyapunov exponent. However, a precise computation of the Lyapunov exponent requires to solve a system of $n(n+1)$ ordinary differential equations; thus solving such a maximization problem would be very time-consuming.

We propose here to use an auxiliary function $f(R, A)$, the computation if which is about 100 times faster than the computation of the Lyapunov exponent over the same time period. It is defined as follows: let $N(0)=\left[\begin{array}{llll}0.1 & 0.1 & 0.1 & 0.1\end{array}\right]$, and $N(t)$ the solution of (1), associated with $N(0)$ and $(R, A)$. Let $\left(\mathcal{R}^{*}, \mathcal{A}^{*}\right)$ be the parameters found in (Vano et al., 2006) and leading to chaotic solutions, and $N^{*}(t)$ be the associated solution of $(1)$, with $N^{*}(0)=N(0)$. We set:

$$
f(R, A)=\delta_{T}(N)\left(1+\min \left\{\frac{\sigma_{\frac{T}{2}, T}(N)}{\sigma_{\frac{T}{2}, T}\left(N^{*}\right)}, 1\right\} \times \min \left\{\frac{\gamma_{\frac{T}{2}, T}(N)}{\gamma_{\frac{T}{2}, T}\left(N^{*}\right)}, 2\right\}\right),
$$

where $\delta_{T}(N)=1$ if $N_{i}(t)>\varepsilon$ for all $i=1 \ldots n$ and $t \in(0, T)$, and $\delta_{T}(N)=\min _{i=1 \ldots n, t \in(0, T)} \frac{N_{i}(t)}{\varepsilon}$ if this condition is not fulfilled. The function $\sigma_{\frac{T}{2}, T}$ associates to a continuous function $X$ : $\mathbb{R}_{+} \rightarrow \mathbb{R}^{n}$, the average standard deviation:

$$
\sigma_{\frac{T}{2}, T}(X)=\frac{1}{n} \sum_{i=1}^{n} \sqrt{\int_{\frac{T}{2}}^{T} X_{i}^{2}(t) d t-\left(\int_{\frac{T}{2}}^{T} X_{i}(t) d t\right)^{2}},
$$

and $\gamma_{\frac{T}{2}, T}$ measures the average total variation of the power spectrum:

$$
\gamma_{\frac{T}{2}, T}(X)=\frac{1}{n} \sum_{i=1}^{n} \int_{0}^{+\infty}\left|\frac{\partial}{\partial \omega}\left(\frac{S\left(X_{i}, \omega\right)}{\max _{\zeta>0} S\left(X_{i}, \zeta\right)}\right)\right| d \omega
$$

with:

$$
S\left(X_{j}, \omega\right)=\frac{2}{T}\left|\int_{\frac{T}{2}}^{T} e^{i \omega\left(t-\frac{T}{2}\right)} X_{j}(t) d t\right|^{2}, \text { for } j=1 \ldots n .
$$

Thus, values of $\delta_{T}$ close to 1 are associated with trajectories far from 0 (we took $\varepsilon=2.10^{-4}$ for our computations), high values of $\sigma_{\frac{T}{2}, T}$ are associated with highly variable functions, and high values of $\gamma_{\frac{T}{2}, T}$ exclude periodic and quasi-periodic solutions (see Eckmann and Ruelle 
1985). The final time $T$ shall be chosen large enough, such that transitory regimes are not taken into account.

We build a sequence $\left(R_{k}, A_{k}\right)$ of elements of $E:=\left\{\left(\mathbb{R}_{+}^{*}\right)^{n} \times \mathcal{M}_{n}\left(\mathbb{R}_{+}\right)\right\} \cap\left\{0<r_{i}<5,0 \leq\right.$ $\left.a_{i j}<5, i, j=1 \ldots n, i \neq j\right\} \cap\left\{a_{i i}=1, i=1 \ldots n\right\}$, with the following simulated annealing algorithm (SA):

Initialize $\left(R_{0}, A_{0}\right)$

do

Choose randomly a neighbour $\widetilde{(R, A)}$ of $\left(R_{k}, A_{k}\right)$

Draw randomly $w \in(0,1)$ in a uniform law

if $w<e^{\frac{f(\widetilde{R, A})-f\left(R_{k}, A_{k}\right)}{\Theta(k)}}$

$\left(R_{k+1}, A_{k+1}\right) \leftarrow \widetilde{(R, A)}$

else

$\left(R_{k+1}, A_{k+1}\right) \leftarrow\left(R_{k}, A_{k}\right)$

\section{endif}

$k \leftarrow k+1$

if $f\left(R_{k}, A_{k}\right)>\max _{j<k} f\left(R_{j}, A_{j}\right)$

Compute the Lyapunov exponent associated to $\left(R_{k}, A_{k}\right)$

\section{endif}

loop

The sequence $\Theta(n)$ (cooling schedule) is composed of real positive numbers, decreasing to 0 . The simulated annealing algorithm gives a sequence $\left(R_{k}, A_{k}\right)$ of elements of $E$. It is proved (see e.g. Hajek 1988; Belisle 1992) that, for a cooling schedule $\Theta(k)$ which converges sufficiently slowly to 0 , the sequence $\left(R_{k}, A_{k}\right)$ asymptotically converges to a global maximum $\widehat{(R, A)}$ of the function $f$ (but see remark below). In our computations, we used $\Theta(k)=\theta_{0} \times 0.9999^{k}$ for the cooling schedule. Two elements of $E$ were defined as neighbours if and only if they differed by one component. The algorithm led to positive Lyapunov exponents in approximately one over five computations.

Taking a low initial temperature $\theta_{0}=1$, and $\left(R_{0}, A_{0}\right)=\left(\mathcal{R}^{*}, \mathcal{A}^{*}\right)$ the parameters found by Vano et al. (2006), we obtained the couple $\left(\mathcal{R}_{1}, \mathcal{A}_{1}\right)$ :

$$
\mathcal{A}_{1}=\left[\begin{array}{llll}
1 & 2.419 & 2.248 & 0.0023 \\
0.001 & 1 & 0.001 & 1.3142 \\
2.3818 & 0.001 & 1 & 0.4744 \\
1.21 & 0.5244 & 0.001 & 1
\end{array}\right], \mathcal{R}_{1}=\left[\begin{array}{l}
1.7741 \\
1.0971 \\
1.5466 \\
4.4116
\end{array}\right]
$$

The couples $\left(\mathcal{R}_{2}, \mathcal{A}_{2}\right)$ and $\left(\mathcal{R}_{3}, \mathcal{A}_{3}\right)$ were obtained using $\theta_{0}=300$, and starting from random initial guesses $\left(R_{0}, A_{0}\right)$ :

$$
\mathcal{A}_{2}=\left[\begin{array}{llll}
1 & 0.3064 & 2.9141 & 0.8668 \\
0.125 & 1 & 0.3346 & 1.854 \\
1.9833 & 3.5183 & 1 & 0.001 \\
0.6986 & 0.9653 & 2.1232 & 1
\end{array}\right], \mathcal{R}_{2}=\left[\begin{array}{l}
1 \\
0.1358 \\
1.4936 \\
4.8486
\end{array}\right],
$$


and

$$
\mathcal{A}_{3}=\left[\begin{array}{llll}
1 & 3.6981 & 1.4368 & 0.0365 \\
0 & 1 & 1.7781 & 3.7306 \\
0.5271 & 4.1593 & 1 & 1.3645 \\
0.8899 & 0.2127 & 3.4711 & 1
\end{array}\right], \mathcal{R}_{3}=\left[\begin{array}{l}
4.4208 \\
0.8150 \\
4.5068 \\
1.4172
\end{array}\right] .
$$

The corresponding values of the auxiliary function $f$ (computed at $T=4000$ ) and of the Lyapunov exponent (computed at $T=10^{4}$ ) are: $f\left(\mathcal{R}_{1}, \mathcal{A}_{1}\right)=1.4$ and $\lambda_{1}=0.04, f\left(\mathcal{R}_{2}, \mathcal{A}_{2}\right)=$ 0.8 and $\lambda_{2}=0.01, f\left(\mathcal{R}_{3}, \mathcal{A}_{3}\right)=1.2$ and $\lambda_{3}=0.03, f\left(\mathcal{R}^{*}, \mathcal{A}^{*}\right)=1$ and $\lambda^{*}=0.02$.

Remark The cooling rate $\Theta(k)$ leading to the global maximum with probability 1 decreases very slowly (logarithmically) and cannot be used in practice; see Henderson et al. (2003) for a detailed discussion. Empirically, a good trade-off between quality of solutions and time required for computation is obtained with exponential cooling schedules of the type $\Theta(k)=\Theta_{0} \times \alpha^{k}$, with $\alpha<1$, first proposed by Kirkpatrick et al. (1983). Many other cooling schedules are possible, but too rapid cooling results in a system frozen into a state far from the optimal one. The starting temperature $\Theta_{0}$ should be chosen high enough to initially accept all changes $\left(R_{k+1}, A_{k+1}\right) \leftarrow \widetilde{(R, A)}$, whatever the neighbour $\widetilde{(R, A)}$.

For this type of algorithm, there are no general rules for the choice of the stopping criterion (see Henderson et al. 2003), which should be heuristically adapted to the considered optimization problem.

\section{Appendix B: equations of $\Delta_{1}, \Delta_{2}$ and $\Delta_{3}$}

The equations of the three segments in Fig. 3 are $\Delta_{1}=\{\tau(0.02,-0.007)+(1-\tau)(0.02,0.06)\}$, $\Delta_{2}=\{\tau(0.9,0.005)+(1-\tau)(1.01,0.005)\}$ and $\Delta_{3}=\{\tau(0.03,0.96)+(1-\tau)(0.03,1)\}$, for $\tau \in(0,1)$.

\section{References}

Anosov, D.V., 1963. Ergodic properties of geodesic flows on closed Riemannian manifolds of negative curvature. Soviet Mathematics, Doklady 4, 1153-1156.

Armstrong, R., McGehee, R., 1980. Competitive exclusion. Am. Nat. 15, 151-170.

Arnéodo, A., Coullet, P., Peyraud, J., Tresser, C., 1982. Strange attractors in Volterra equations for species in competition. J. Math. Biol. 14, 153-157.

Arnol'd, V., 1966. Sur la géométrie différentielle des groupes de Lie de dimension infinie et ses applications à l'hydrodynamique des fluides parfaits. Ann. I. Fourier 16, 319-361.

Belisle, C.J.P., 1992. Convergence theorems for a class of simulated annealing algorithms on $\mathbb{R}^{d}$. J. Appl. Probab. 29, 885-892.

Bell, G., 2001. Neutral Macroecology. Science 293, 2413-2418.

Benincà, E., Huisman, J., Heerkloss, R., Jöhnk, K.D., Branco, P., Van Nes, E.H., Scheffer, M., Ellner, S.P., 2008. Chaos in a long-term experiment with a plankton community. Nature 451, 822-825.

Cantrell R.S., Cosner, C., 2003. Spatial Ecology via Reaction-Diffusion Equations. Ser. Math. Comput. Biol., Chichester, UK: John Wiley and Sons.

Costantino, R.F., Desharnais, R.A., Cushing, J.M., Dennis, B., 1997. Chaotic dynamics in an insect population. Science 275, 389-391. 
Durrett, R. and Levin, S., 1994. The importance of being discrete (and spatial). Theor. Popul. Biol. 46, 363-394.

Eckmann, J.-P., Ruelle, D., 1985. Ergodic theory of chaos and strange attractors. Rev. Mod. Phys. 57, 617-656.

Ellner, S., Turchin, P. 1995 Chaos in a noisy world: new methods and evidence from time-series analysis. Am. Nat. 145, 343-375.

Ghil, M., Chekroun, M.D., Simonnet, E., 2008. Climate dynamics and fluid mechanics: Natural variability and related uncertainties. Physica D 237, 2111-2126.

Gilpin, M.E., 1975. Limit cycles in competition communities. Am. Nat. 109, 51-60.

Grebogi, C., Ott, E., Yorke, J.A., 1987. Chaos, strange attractors, and fractal basin boundaries in nonlinear dynamics. Science 238, 632-638.

Hajek, B., 1988. Cooling schedules for optimal annealing. Math. Method. Oper. Res. 13, 311-329.

Hanski, I.A. and Gilpin, M.E., 1996. Metapopulation Biology: Ecology, Genetics, and Evolution. Academic press, NY.

Hardin, G., 1960. The competitive exclusion principle. Science 131, 1291-1297.

Henderson, D., Jacobson, S.H., Johnson A.W., 2003. The Theory and Practice of Simulated Annealing. In: Glover, F., Kochenberger, G. (Eds.) Handbook on Metaheuristics. Norwell, MA: Kluwer Academic Publishers, pp. 287-319.

Hirsch, M.W., 1982. Systems of differential equations which are competitive or cooperative I: Limit sets. SIAM J. Appl. Math. 13, 167-179.

Hirsch, M.W., 1985. Systems of differential equations which are competitive and cooperative II: Convergence almost everywhere. SIAM J. Math. Anal. 16, 423-439.

Hirsch, M.W., 1988. Systems of differential equations which are competitive or cooperative III: Competing species. Nonlinearity 1, 51-71.

Hirsch, M.W., 1989. Systems of differential equations that are competitive or cooperative. V. Convergence in 3-dimensional systems. J. Differ. Equations 80, 94-106.

Hirsch, M.W., 1990. Systems of differential equations that are competitive or cooperative. IV: Structural stability in three dimensional systems. SIAM J. Math. Anal. 21, 1225-1234.

Hirsch, M.W., 1991. Systems of differential equations that are competitive or cooperative. VI. A local $C^{r}$ closing lemma for 3-dimensional systems. Ergod. Theor. Dyn. Syst. 11, 443-454.

Hubbell, S.P., 2001. The Unified Neutral Theory of Biodiversity and Biogeography. Monographs in Population Biology, Princeton: Princeton University Press.

Huisman, J., Weissing F.J., 1999. Biodiversity of plankton by species oscillations and chaos. Nature 402, 407-410.

Huisman J., Johansson, A.M., Folmer, E.O., Weissing, F.J., 2001. Towards a solution of the plankton paradox: the importance of physiology and life history. Ecol. Lett. 4, 408-411.

Huisman, J., Weissing, F.J., 2001a. Fundamental unpredictability in multispecies competition. Am. Nat. 157, 488-494.

Huisman, J., Weissing, F.J., 2001b. Biological conditions for oscillations and chaos generated by multispecies competition. Ecology 82, 2682-2695.

Hutchinson, G.E., 1961. The Paradox of the Plankton. Am. Nat. 95, 137-145.

Kirkpatrick, S., Gelatt, C.D., Vecchi, M.P., 1983. Optimization by Simulated Annealing. Science 220, 671-680. 
Lande, R., 1993. Risks of population extinction from demographic and environmental stochasticity and random catastrophes. Am. Nat. 142, 911-927.

Levin, S.A., 1970. Community equilibria and stability, and an extension of the competitive exclusion principle. Am. Nat. 104, 413-423.

Levins, R., 1969. Some demographic and genetic consequences of environmental heterogeneity for biological control. Bull. Entomol. Soc. Am. 15, 237-240.

Lorenz, E.N., 1963. Deterministic nonperiodic flow. J. Atmos. Sci. 20, 130-141.

Lotka, A.J., 1925. Elements of Physical Biology. Williams and Wilkins (Baltimore). Reprinted 1956, NY: Dover Press.

Lotka, A.J., 1932. The growth of mixed populations: two species competing for a common food supply. Journal of the Washington Academy of Sciences 22, 461-469.

MacArthur, R.H., Levins, R., 1967. The limiting similarity, convergence, and divergences of coexisting species. Am. Nat. 101, 377-385.

May, R.M., 1974. Stability and Complexity in Model Ecosystems. Cambridge University Press.

May R.M., Leonard, J.W., 1975. Nonlinear aspects of competition between three species. SIAM J. Appl. Math. 29, 243-253.

Murray, J.D., 2002. Mathematical biology. Third edition, Interdisciplinary Applied Mathematics 17, NY: Springer-Verlag.

Nese, J.M., 1989. Quantifying local predictability in phase space. Physica D 35, 237-250.

Oksanen, L., Oksanen, T., 1992. Long-term microtine dynamics in North Fennoscandian tundra: the vole cycle and the lemming chaos. Ecography 15, 226-236.

Petrovskii, S.V., Malchow, H., 2001. Spatio-temporal chaos in an ecological community as a response to unfavourable environmental changes. Adv. Complex Syst. 4, 227-249.

Poincaré, H., 1892. Les méthodes nouvelles de la mécanique céleste. Paris: Gauthier-Villars.

Ruelle, D., Takens, F., 1971. On the nature of turbulence. Commun. Math. Phys. 20, 167-192.

Shaw, R., 1981. Strange attractors, chaotic behavior, and information flow. Z. Naturforsch. A 36, 80-112.

Smale, S., 1967. Differential dynamical systems. B. Am. Math. Soc. 73, 747-817.

Smale, S., 1976. On the differential equations of species in competition. J. Math. Biol. 3, 5-7.

Turchin, P., Ellner, S.P., 2000. Living on the edge of chaos: population dynamics of Fennoscandian voles. Ecology 81, 3099-3116.

Turchin, P. 2003 Complex Population Dynamics: a Theoretical/Empirical Synthesis. Princeton University Press.

Vano, J.A., Wildenberg, J.C., Anderson, M.B., Noel, J.K., Sprott, J.C., 2006. Chaos in low-dimensional LotkaVolterra models of competition. Nonlinearity 19, 2391-2404.

Volterra, V., 1931. Leçons sur la Théorie Mathématique de la Lutte pour la Vie. Paris: Marcel Brelot. 